\title{
Inverse source problems for eddy current equations
}

\author{
Ana Alonso Rodríguez ${ }^{1}$, Jessika Camaño ${ }^{2}$ and Alberto Valli ${ }^{1}$ \\ ${ }^{1}$ Department of Mathematics, University of Trento, Italy. ${ }^{2}$ Department of \\ Engineering Mathematics, University of Concepción, Chile. \\ E-mail: alonso@science.unitn.it, jcamano@ing-mat.udec.cl, \\ valli@science.unitn.it
}

\begin{abstract}
We study the inverse source problem for the eddy current approximation of Maxwell equations. As for the full system of Maxwell equations, we show that a volume current source cannot be uniquely identified by the knowledge of the tangential components of the electromagnetic fields on the boundary, and we characterize the space of non-radiating sources. On the other hand, we prove that the inverse source problem has a unique solution if the source is supported on the boundary of a subdomain or if it is the sum of a finite number of dipoles. We address the applicability of this result for the localization of brain activity from electroencephalography and magnetoencephalography measurements.
\end{abstract}

\section{Introduction}

Electroencephalography (EEG) and magnetoencephalography (MEG) are two noninvasive techniques used to localize electric activity in the brain from measurements of external electromagnetic signals. EEG measures the scalp electric potential, while MEG measures the external magnetic flux. From the mathematical point of view the goal is to solve an inverse problem for determining the source current distribution in a heterogeneous media from boundary measurements of the fields.

The frequency spectrum for electrophysiological signals in EEG and MEG is typically below $1000 \mathrm{~Hz}$, most frequently between 0.1 and $100 \mathrm{~Hz}$. For this reason most theoretical works on biomedical applications focus on the static approximation of the Maxwell equations, in which the time variation of both electric and magnetic fields is disregarded.

Recently He and Romanov [1], Ammari et al. [2] and Albanese and Monk [3] investigate the localization of brain activity through the inverse source problem for the full Maxwell system of equations. In this paper we analyze the inverse source problem for an alternative model: the eddy current (or low frequency approximation) of Maxwell equations. In the eddy current model the time variation of the electric field is disregarded, while time variation of the magnetic field is kept. 
Let us consider electromagnetic phenomena at frequency $\omega \neq 0$. The time-harmonic full Maxwell system of equations read

$$
\begin{array}{ll}
\operatorname{curl} \mathbf{H}-i \omega \epsilon \mathbf{E}=\sigma \mathbf{E}+\mathbf{J}_{e} & \text { (Maxwell-Ampère equation) } \\
\operatorname{curl} \mathbf{E}+i \omega \mu \mathbf{H}=\mathbf{0} & \text { (Faraday equation). }
\end{array}
$$

Here $\mathbf{E}, \mathbf{H}$ denote the electric and magnetic fields, respectively; $\mathbf{J}_{e}$ is the applied current density; $\epsilon$ is the electric permittivity, $\mu$ the magnetic permeability and $\sigma$ the electric conductivity.

The eddy current model is formally obtained by neglecting the displacement current term:

$$
\begin{aligned}
& \operatorname{curl} \mathbf{H}=\sigma \mathbf{E}+\mathbf{J}_{e} \\
& \operatorname{curl} \mathbf{E}+i \omega \mu \mathbf{H}=\mathbf{0} .
\end{aligned}
$$

Let us consider a conductor $\Omega_{C} \subset \mathbb{R}^{3}$, say, the human head. We assume that $\Omega_{C}$ is a bounded domain with a Lipschitz and connected boundary $\Gamma$. In $\Omega_{C}$ the conductivity $\sigma$ is a symmetric and uniformly positive definite matrix with entries in $L^{\infty}\left(\Omega_{C}\right)$. We consider also a computational domain $\Omega \subset \mathbb{R}^{3}$, say, the room where the problem is studied. We assume that $\Omega$ is a bounded simply-connected domain, completely containing $\Omega_{C}$ and with Lipschitz boundary $\partial \Omega$. Moreover we assume that $\Omega_{I}:=\Omega \backslash \overline{\Omega_{C}}$ is connected. $\Omega_{I}$ is an insulator, the air surrounding the head, hence $\sigma$ is vanishing in $\Omega_{I}$. We also assume that the electric permeability $\mu$ and the electric permittivity $\epsilon$ are symmetric and uniformly positive definite matrices with entries in $L^{\infty}(\Omega)$.

On the boundary $\partial \Omega$ we can impose magnetic boundary condition $\mathbf{H} \times \mathbf{n}=\mathbf{0}$ on $\partial \Omega$ or electric boundary condition $\mathbf{E} \times \mathbf{n}=\mathbf{0}$ on $\partial \Omega$. (Here $\mathbf{n}$ denotes the unit outward normal vector on $\partial \Omega$.)

Since $\sigma$ is equal zero in insulators, equations (2) do not completely determine the electric field in $\Omega_{I}$. In that region one has to add $\operatorname{div}(\epsilon \mathbf{E})=0$ because there are no charges in an insulator. This is a "gauge" condition necessary for having uniqueness. When imposing magnetic boundary condition the additional "gauge" condition $\epsilon \mathbf{E} \cdot \mathbf{n}=0$ on $\partial \Omega$ is also necessary.

From Faraday law $\mu^{-1} \operatorname{curl} \mathbf{E}=-i \omega \mathbf{H}$ and inserting this result in Ampère law one has $\operatorname{curl}\left(\mu^{-1} \operatorname{curl} \mathbf{E}\right)=-i \omega\left(\sigma \mathbf{E}+\mathbf{J}_{e}\right)$. So the $\mathbf{E}$-based formulation of the eddy current model reads

$$
\begin{cases}\operatorname{curl}\left(\mu^{-1} \operatorname{curl} \mathbf{E}\right)+i \omega \sigma \mathbf{E}=-i \omega \mathbf{J}_{e} & \text { in } \Omega \\ \operatorname{div}(\epsilon \mathbf{E})=0 & \text { in } \Omega_{I} \\ \left(\mu^{-1} \operatorname{curl} \mathbf{E}\right) \times \mathbf{n}=\mathbf{0} & \text { on } \partial \Omega \\ \epsilon \mathbf{E} \cdot \mathbf{n}=0 & \text { on } \partial \Omega\end{cases}
$$

for the magnetic boundary condition, and

$$
\begin{cases}\operatorname{curl}\left(\mu^{-1} \operatorname{curl} \mathbf{E}\right)+i \omega \sigma \mathbf{E}=-i \omega \mathbf{J}_{e} & \text { in } \Omega \\ \operatorname{div}(\epsilon \mathbf{E})=0 & \text { in } \Omega_{I} \\ \mathbf{E} \times \mathbf{n}=\mathbf{0} & \text { on } \partial \Omega\end{cases}
$$

for the electric boundary condition. In this paper we will focus on problem (3); the same results can be proved for problem (4). 
In the static approximation also the time variation of the magnetic field is disregarded, thus one has:

$$
\begin{aligned}
& \operatorname{curl} \mathbf{H}=\sigma \mathbf{E}+\mathbf{J}_{e} \\
& \operatorname{curl} \mathbf{E}=\mathbf{0}
\end{aligned}
$$

(where $\mathbf{J}_{e}$ can still depend on time, which has to be regarded as a parameter). From the second equation in (5) the electric field is the gradient of a scalar potential $\mathbf{E}=-\operatorname{grad} V$ and then from the first equation in (5) we obtain $\operatorname{div}(\sigma \operatorname{grad} V)=\operatorname{div} \mathbf{J}_{e}$ in $\Omega_{C}$. On the other hand $\sigma \mathbf{E}+\mathbf{J}_{e}$ is divergence free in $\Omega$, hence we have $\left(\sigma \operatorname{grad} V-\mathbf{J}_{e \mid \Omega_{C}}\right) \cdot \mathbf{n}_{\Gamma}=$ $-\mathbf{J}_{e \mid \Omega_{I}} \cdot \mathbf{n}_{\Gamma}$ on $\Gamma, \mathbf{n}_{\Gamma}$ being the unit normal vector on $\Gamma$ pointing outwards $\Omega_{C}$. Since we are interested in electric sources located in the conductor, namely, $\operatorname{supp} \mathbf{J}_{e} \subset \Omega_{C}$, the boundary condition for the static approximation is the homogeneous Neumann boundary condition $\sigma \operatorname{grad} V \cdot \mathbf{n}_{\Gamma}=0$ on $\Gamma$. The static problem thus reads

$$
\begin{cases}\operatorname{div}(\sigma \operatorname{grad} V)=\operatorname{div} \mathbf{J}_{e} & \text { in } \Omega_{C} \\ \sigma \operatorname{grad} V \cdot \mathbf{n}_{\Gamma}=0 & \text { on } \Gamma,\end{cases}
$$

and the related magnetic field is computed in terms of the primary current $\mathbf{J}_{e}$ and the return current $\sigma \mathbf{E}=-\sigma \operatorname{grad} V$ using the Biot-Savart law in $\mathbb{R}^{3}$ :

$$
\mathbf{H}(\mathbf{x})=\frac{1}{4 \pi} \int_{\Omega_{C}}\left[\mathbf{J}_{e}(\mathbf{y})-\sigma \operatorname{grad} V(\mathbf{y})\right] \times \frac{\mathbf{x}-\mathbf{y}}{|\mathbf{x}-\mathbf{y}|^{3}} d \mathbf{y} .
$$

The inverse source problem consists in the determination of the current source $\mathbf{J}_{e}$ from boundary measurements of the electromagnetic fields. It is well-known since Helmholtz that in general this problem has not a unique solution. For instance, if the source is a radial dipole the magnetic field given by (7) vanishes outside a spherical conductor $\Omega_{C}$ (see, e.g., Sarvas [4]), hence when using the static model the knowledge of the magnetic field on $\Gamma$ does not contribute to the localization of radial dipoles.

The characterization of the source currents that can be reconstructed from suitable measurements on the boundary is not an easy task and depends on the model considered. For the static model in Kress et al. [5] the authors prove that the Biot-Savart operator has a non-trivial null space. Fokas et al. [6] characterize which part of a volume current source in a spherical conductor can be reconstructed from the knowledge of the magnetic field on the boundary. In the same framework, Dassios and Hadjiloizi [7] determine which part of the source can be reconstructed from the electric potential. Instead, concerning dipole sources, He and Romanov [1] show that the measurement of the electric potential on the boundary of the conductor is enough for identifying their location and polarization; a similar result is given in El Badia and Nara [8], assuming the knowledge of the tangential component of the electric field only in a part of the boundary.

When considering the full Maxwell system the existence of non-radiating sources has been proved in Bleistein and Cohen [9]. On the other hand, He and Romanov [1] show that the location and the polarization of a current dipole in a conducting object can be uniquely determined by measuring at a fixed frequency the magnetic field and its 
normal derivative on the whole surface. The same result has been obtained by Ammari et al. [2] from the knowledge of the tangential component of either the electric or the magnetic field on $\Gamma$. Albanese and Monk [3] have characterized which part of a volume source confined in $\Omega_{C}$ can be uniquely identified from measurements of the tangential component of the electric field on $\Gamma$. Moreover, they also prove uniqueness of the inverse source problem if the source is supported on the surface of a-priori known subdomain contained in $\Omega_{C}$ or if it is the sum a finite number of dipole sources. In the last case the tangential component of the electric field uniquely determines the number, position and polarization of the dipoles.

The aim of this paper is to study the uniqueness of the solution of the inverse source problem for the eddy current approximation of Maxwell equations, mainly following the approach proposed by Albanese and Monk [3] for the full Maxwell system of equations. The outline of the paper is as follows. Section 2 is devoted to volume source currents. We prove that when looking for $\mathbf{J}_{e} \in\left(L^{2}\left(\Omega_{C}\right)\right)^{3}$ the inverse problem has not a unique solution and we characterize the space of non-radiating sources. In Section 3 we obtain the uniqueness result for a source current supported on the boundary of a subdomain of $\Omega_{C}$ : it is worth noting that the support of the surface source is not assumed to be known, but it is uniquely determined from the boundary data. In Section 4 we consider the case of dipole sources. First we study the well-posedness of the direct problem, that is, the existence and uniqueness of the solution for the eddy current model assuming that the source is a finite sum of dipoles. Then we prove uniqueness of the inverse source problem, determining the number, the location and the polariziation of the dipole source. We present also an algebraic algorithm for the determination of a dipole source assuming that the tangential component of the electric field on $\Gamma$ is known. In the last section we study how to recover the tangential component of the electric field on $\Gamma$, the data that we use in the inverse problem, from the data that are measured in magnetoenecephalography and electroencephalography.

To conclude this section let us introduce some notation that will be used in the following. The space $H(\operatorname{curl} ; \Omega)$ indicates the set of real or complex vector valued functions $\mathbf{v} \in\left(L^{2}(\Omega)\right)^{3}$ such that curl $\mathbf{v} \in\left(L^{2}(\Omega)\right)^{3}$. We also use the spaces $H^{-1 / 2}\left(\operatorname{curl}_{\tau} ; \Gamma\right):=\left\{\left(\mathbf{n}_{\Gamma} \times \mathbf{v} \times \mathbf{n}_{\Gamma}\right)_{\mid \Gamma} \mid \mathbf{v} \in H\left(\operatorname{curl} ; \Omega_{C}\right)\right\}$ and $H^{-1 / 2}\left(\operatorname{div}_{\tau} ; \Gamma\right):=$ $\left\{\left(\mathbf{v} \times \mathbf{n}_{\Gamma}\right)_{\mid \Gamma} \mid \mathbf{v} \in H\left(\operatorname{curl} ; \Omega_{C}\right)\right\}$. These two spaces are in duality and the following formula of integration by parts holds true:

$$
\int_{\Omega_{C}}(\mathbf{w} \cdot \operatorname{curl} \overline{\mathbf{v}}-\operatorname{curl} \mathbf{w} \cdot \overline{\mathbf{v}})=\int_{\Gamma}\left(\mathbf{w} \times \mathbf{n}_{\Gamma}\right) \cdot \overline{\mathbf{v}} \quad \forall \mathbf{w}, \mathbf{v} \in H\left(\operatorname{curl} ; \Omega_{C}\right) .
$$

The last integral is indeed the duality paring between $\mathbf{w} \times \mathbf{n}_{\Gamma} \in H^{-1 / 2}\left(\operatorname{div}_{\tau} ; \Gamma\right)$ and $\mathbf{n}_{\Gamma} \times \mathbf{v} \times \mathbf{n}_{\Gamma} \in H^{-1 / 2}\left(\operatorname{curl}_{\tau} ; \Gamma\right)$.

\section{Non-uniqueness of volume currents}

In this section we investigate the uniqueness of the inverse source problem assuming that the unknown source $\mathbf{J}_{e}$ is a function in $\left(L^{2}\left(\Omega_{C}\right)\right)^{3}$. First we will prove that without 
additional information, the source cannot be reconstructed from the knowledge of the tangential component of the electric field on $\Gamma$. We then characterize the space of nonradiating sources (those sources in $\left(L^{2}\left(\Omega_{C}\right)\right)^{3}$ that generate an electric field normal to the surface $\Gamma$ ) and prove that sources $\mathbf{J}_{e} \in\left(L^{2}\left(\Omega_{C}\right)\right)^{3}$ that are orthogonal to the space of non-radiating sources are uniquely determined by the tangential component on $\Gamma$ of the electric field. The result is analogous to the one obtained by Albanese and Monk [3] for the full Maxwell system.

If $\mathbf{J}_{e} \in\left(L^{2}\left(\Omega_{C}\right)\right)^{3}$ it is known that problems (3) has a unique solution $\mathbf{E}$ and the magnetic field can be computed from Faraday law: $\mathbf{H}=-(i \omega \mu)^{-1} \operatorname{curl} \mathbf{E}$ in $\Omega$.

Multiplying the first equation in (3) by a regular enough test function $\mathbf{z}$, integration by parts in $\Omega_{C}$ easily yields

$$
-i \omega \int_{\Omega_{C}} \mathbf{J}_{e} \cdot \overline{\mathbf{z}}=\int_{\Omega_{C}} \mathbf{E} \cdot\left[\operatorname{curl}\left(\mu^{-1} \operatorname{curl} \overline{\mathbf{z}}\right)+i \omega \sigma \overline{\mathbf{z}}\right]-\int_{\Gamma}\left[\mathbf{E} \times \mathbf{n}_{\Gamma} \cdot\left(\mu^{-1} \operatorname{curl} \overline{\mathbf{z}}\right)-i \omega \mathbf{H} \times \mathbf{n}_{\Gamma} \cdot \overline{\mathbf{z}}\right] .
$$

Therefore, if $\mathbf{z} \in H\left(\operatorname{curl} ; \Omega_{C}\right)$ is such that

$$
\operatorname{curl}\left(\mu^{-1} \operatorname{curl} \mathbf{z}\right)-i \omega \sigma \mathbf{z}=\mathbf{0} \text { in } \Omega_{C},
$$

the current density $\mathbf{J}_{e}$ satisfies the representation formula

$$
\int_{\Omega_{C}} \mathbf{J}_{e} \cdot \overline{\mathbf{z}}=(i \omega)^{-1} \int_{\Gamma} \mathbf{E} \times \mathbf{n}_{\Gamma} \cdot\left(\mu^{-1} \operatorname{curl} \overline{\mathbf{z}}\right)-\int_{\Gamma} \mathbf{H} \times \mathbf{n}_{\Gamma} \cdot \overline{\mathbf{z}} .
$$

The right hand term in (8) has been called reciprocity functional, taking the name from the Lorentz reciprocity principle in electromagnetism, or else the Maxwell-Betti reciprocity principle in elastostatics (see, e.g., Andrieux and Ben Abda [10], El Badia and Ha-Duong [11]). It is often used in the analysis of inverse source problems (see, e.g., Novikov [12], Isakov [13]).

Let us define

$$
\mathcal{W}=\left\{\mathbf{z} \in H\left(\operatorname{curl} ; \Omega_{C}\right) \mid \operatorname{curl}\left(\mu^{-1} \operatorname{curl} \mathbf{z}\right)-i \omega \sigma \mathbf{z}=\mathbf{0} \text { in } \Omega_{C}\right\} .
$$

It is clear that $\mathcal{W}$ is not a trivial subspace of $\left(L^{2}\left(\Omega_{C}\right)\right)^{3}$ : since both $\mu$ and $\sigma$ are bounded and uniformly positive definite in $\Omega$, for each $\xi \in H_{\operatorname{div}, \tau}^{-1 / 2}(\Gamma)$ there exists a unique $\mathbf{u}(\xi) \in H\left(\operatorname{curl} ; \Omega_{C}\right)$ such that $\mathbf{u}(\xi) \in \mathcal{W}$ and $\mathbf{u}(\xi) \times \mathbf{n}_{\Gamma}=\xi$ on $\Gamma$.

Denoting by $W$ the closure of $\mathcal{W}$ in $\left(L^{2}\left(\Omega_{C}\right)\right)^{3}$ we have the orthogonal splitting

$$
\left(L^{2}\left(\Omega_{C}\right)\right)^{3}=W \oplus W^{\perp} .
$$

Lemma 2.1 Consider $\boldsymbol{\eta} \in\left(C_{0}^{\infty}\left(\Omega_{C}\right)\right)^{3}$ and set $\boldsymbol{\phi}=\operatorname{curl}\left(\mu^{-1} \operatorname{curl} \boldsymbol{\eta}\right)+i \omega \sigma \boldsymbol{\eta}$. Then $\phi \in W^{\perp}$ (and $W^{\perp}$ is not a trivial subspace).

Proof. Take $\mathbf{z} \in \mathcal{W}$. Then

$$
\begin{aligned}
\int_{\Omega_{C}} \boldsymbol{\phi} \cdot \overline{\mathbf{z}} & =\int_{\Omega_{C}}\left[\operatorname{curl}\left(\mu^{-1} \operatorname{curl} \boldsymbol{\eta}\right)+i \omega \sigma \boldsymbol{\eta}\right] \cdot \overline{\mathbf{z}} \\
& =\int_{\Omega_{C}} \boldsymbol{\eta} \cdot\left[\operatorname{curl}\left(\mu^{-1} \operatorname{curl} \overline{\mathbf{z}}\right)+i \omega \sigma \overline{\mathbf{z}}\right]=0
\end{aligned}
$$

and a density argument shows that $\phi \in W^{\perp}$. 
Note that, if $\boldsymbol{\eta}$ is a non-vanishing real vector field, one obtains $\operatorname{Im} \boldsymbol{\phi} \neq \mathbf{0}$, hence $W^{\perp}$ is not a trivial subspace.

Let us split the current density $\mathbf{J}_{e}$ as

$$
\mathbf{J}_{e}=\mathbf{J}_{e}^{\sharp}+\mathbf{J}_{e}^{\perp}, \quad \mathbf{J}_{e}^{\sharp} \in W, \mathbf{J}_{e}^{\perp} \in W^{\perp} .
$$

Theorem 2.1 (i) Let us assume that $\mathbf{J}_{e}=\mathbf{J}_{e}^{\sharp} \in W$ and that $\mathbf{E}^{\sharp}$ is the corresponding solution of the eddy current problem. Then the knowledge of $\mathbf{E}^{\sharp} \times \mathbf{n}_{\Gamma}$ on $\Gamma$ uniquely determines $\mathbf{J}_{e}^{\sharp}$.

(ii) Let us assume that $\mathbf{J}_{e}=\mathbf{J}_{e}^{\perp} \in W^{\perp}$ and that $\mathbf{E}^{\perp}$ is the corresponding solution of the eddy current problem. Then $\mathbf{E}^{\perp} \times \mathbf{n}_{\Gamma}=\mathbf{0}$ and $\mathbf{H}^{\perp} \times \mathbf{n}_{\Gamma}=\mathbf{0}$ on $\Gamma$, namely, $\mathbf{J}_{e}^{\perp}$ is a non-radiating source.

Proof. (i) The electric field in the insulator satisfy

$$
\begin{array}{ll}
\operatorname{curl}\left(\mu^{-1} \operatorname{curl} \mathbf{E}^{\sharp}\right)=\mathbf{0} & \text { in } \Omega_{I} \\
\operatorname{div}\left(\epsilon \mathbf{E}^{\sharp}\right)=0 & \text { in } \Omega_{I} \\
\left(\mu^{-1} \operatorname{curl} \mathbf{E}^{\sharp}\right) \times \mathbf{n}=\mathbf{0} & \text { on } \partial \Omega \\
\epsilon \mathbf{E}^{\sharp} \cdot \mathbf{n}=0 & \text { on } \partial \Omega .
\end{array}
$$

If $\mathbf{E}^{\sharp} \times \mathbf{n}_{\Gamma}=\mathbf{0}$ on $\Gamma$, multiplying the first equation by $\mathbf{E}^{\sharp}$ and integrating by parts one easily finds curl $\mathbf{E}^{\sharp}=\mathbf{0}$, then $\mathbf{E}^{\sharp}=\mathbf{0}$ in $\Omega_{I}$. Consequently, $\mathbf{H}^{\sharp}=-(i \omega \mu)^{-1} \operatorname{curl} \mathbf{E}^{\sharp}=\mathbf{0}$ in $\Omega_{I}$ and in particular $\mathbf{H}^{\sharp} \times \mathbf{n}_{\Gamma}=\mathbf{0}$ on $\Gamma$. Therefore from (8) we know that $\int_{\Omega_{C}} \mathbf{J}_{e}^{\sharp} \cdot \overline{\mathbf{z}}=0$ for each $\mathbf{z} \in \mathcal{W}$, hence, by a density argument, for each $\mathbf{z} \in W$. Taking $\mathbf{z}=\mathbf{J}_{e}^{\sharp} \in W$, the thesis follows.

(ii) Since $\mathbf{J}_{e}^{\perp} \in W^{\perp}$ from (8) we have that for all $\mathbf{z} \in W$

$$
\int_{\Gamma} \mathbf{E}^{\perp} \times \mathbf{n}_{\Gamma} \cdot\left(\mu^{-1} \operatorname{curl} \overline{\mathbf{z}}\right)-i \omega \int_{\Gamma} \mathbf{H}^{\perp} \times \mathbf{n}_{\Gamma} \cdot \overline{\mathbf{z}}=0
$$

For each $\boldsymbol{\eta} \in H_{\operatorname{div}, \tau}^{-1 / 2}(\Gamma)$ we denote by $\mathbf{Z} \in H(\operatorname{curl} ; \Omega)$ the solution to

$$
\begin{cases}\operatorname{curl}\left(\mu^{-1} \operatorname{curl} \mathbf{Z}\right)-i \omega \sigma \mathbf{Z}=\mathbf{0} & \text { in } \Omega_{C} \cup \Omega_{I} \\ \operatorname{div}(\epsilon \mathbf{Z})=0 & \text { in } \Omega_{I} \\ \left(\mu^{-1} \operatorname{curl} \mathbf{Z}\right) \times \mathbf{n}=\mathbf{0} & \text { on } \partial \Omega \\ \epsilon \mathbf{Z} \cdot \mathbf{n}=0 & \text { on } \partial \Omega \\ \left(\mu^{-1} \operatorname{curl} \mathbf{Z}\right)_{\mid \Omega_{C}} \times \mathbf{n}_{\Gamma}=\left(\mu^{-1} \operatorname{curl} \mathbf{Z}\right)_{\mid \Omega_{I}} \times \mathbf{n}_{\Gamma}+\boldsymbol{\eta} & \text { on } \Gamma,\end{cases}
$$

which in weak form reads

$$
\text { find } \mathbf{Z} \in V: \int_{\Omega}\left(\mu^{-1} \operatorname{curl} \mathbf{Z} \cdot \operatorname{curl} \overline{\mathbf{v}}-i \omega \sigma \mathbf{Z} \cdot \overline{\mathbf{v}}\right)=\int_{\Gamma} \eta \cdot \overline{\mathbf{v}} \quad \forall \mathbf{v} \in V
$$

where $V:=\left\{\mathbf{v} \in H(\operatorname{curl} ; \Omega): \operatorname{div}(\epsilon \mathbf{v})=0\right.$ in $\Omega_{I}$ and $\epsilon \mathbf{Z} \cdot \mathbf{n}=0$ on $\left.\partial \Omega\right\}$. It is wellknown that the sesquilinear form at the left hand side is coercive in $V$ (see Alonso Rodríguez and Valli [14], Theorem 2.3), therefore the problem is uniquely solvable.

As a test function in (9) we can thus select $\mathbf{Z}_{\mid \Omega_{C}} \in W$, obtaining

$$
\begin{aligned}
\int_{\Gamma} \mathbf{E}^{\perp} \times \mathbf{n}_{\Gamma} \cdot \mu^{-1} \operatorname{curl} \overline{\mathbf{Z}_{\mid \Omega_{C}}} & =-\int_{\Gamma} \mathbf{E}^{\perp} \cdot \overline{\boldsymbol{\eta}}-\int_{\Gamma} \mathbf{E}^{\perp} \cdot\left(\mu^{-1} \operatorname{curl} \overline{\mathbf{Z}_{\mid \Omega_{I}}} \times \mathbf{n}_{\Gamma}\right) \\
& =-\int_{\Gamma} \mathbf{E}^{\perp} \cdot \overline{\boldsymbol{\eta}}+\int_{\Omega_{I}} \mu^{-1} \operatorname{curl} \mathbf{E}^{\perp} \cdot \operatorname{curl} \overline{\mathbf{Z}_{\mid \Omega_{I}}}
\end{aligned}
$$




$$
\begin{aligned}
-i \omega \int_{\Gamma} \mathbf{H}^{\perp} \times \mathbf{n}_{\Gamma} \cdot \overline{\mathbf{Z}_{\mid \Omega_{C}}} & =-\int_{\Gamma} \mu^{-1} \operatorname{curl} \mathbf{E}^{\perp} \cdot \overline{\mathbf{Z}_{\mid \Omega_{I}}} \times \mathbf{n}_{\Gamma} \\
& =-\int_{\Omega_{I}} \mu^{-1} \operatorname{curl} \mathbf{E}^{\perp} \cdot \operatorname{curl} \overline{\mathbf{Z}_{\mid \Omega_{I}}} .
\end{aligned}
$$

In conclusion, we have found

$$
\int_{\Gamma} \mathbf{E}^{\perp} \cdot \overline{\boldsymbol{\eta}}=0
$$

for each $\boldsymbol{\eta} \in H_{\operatorname{div}, \tau}^{-1 / 2}(\Gamma)$, hence $\mathbf{n}_{\Gamma} \times \mathbf{E}^{\perp} \times \mathbf{n}_{\Gamma}=\mathbf{0}$ on $\Gamma$.

Proceeding as in the proof of (i) we show that $\mathbf{E}^{\perp} \times \mathbf{n}_{\Gamma}=\mathbf{0}$ on $\Gamma$ implies $\mathbf{H}^{\perp} \times \mathbf{n}_{\Gamma}=\mathbf{0}$ on $\Gamma$, and the proof is complete.

\section{Uniqueness of surface currents}

In this section we prove that if the source current is known to be supported on the surface of a subdomain contained in $\Omega_{C}$, then both the surface and the value of the surface current are uniquely determined by the tangential component of the electric field on $\Gamma$. A similar result, but assuming that the surface is a-priori known, has been previously obtained for the full Maxwell systems (see Albanese and Monk [3]).

First, we start by considering a surface current $\mathbf{J}_{*} \in H^{-1 / 2}\left(\operatorname{div}_{\tau} ; \partial B\right)$, where $B$ is an open connected set with Lipschitz boundary $\partial B$ and satisfying $\bar{B} \subset \Omega_{C}$.

The direct problem reads

$$
\begin{cases}\operatorname{curl} \mathbf{E}_{*}+i \omega \mu \mathbf{H}_{*}=\mathbf{0} & \text { in } \Omega \\ \operatorname{curl} \mathbf{H}_{*}=\sigma \mathbf{E}_{*} & \text { in } B \cup(\Omega \backslash \bar{B}) \\ \operatorname{div}\left(\epsilon \mathbf{E}_{*}\right)=0 & \text { in } \Omega_{I} \\ \mathbf{H}_{*} \times \mathbf{n}=\mathbf{0} & \text { on } \partial \Omega \\ \epsilon \mathbf{E}_{*} \cdot \mathbf{n}=0 & \text { on } \partial \Omega \\ \mathbf{H}_{* \mid B} \times \mathbf{n}_{B}-\mathbf{H}_{* \mid \Omega \backslash \bar{B}} \times \mathbf{n}_{B}=\mathbf{J}_{*} & \text { on } \partial B\end{cases}
$$

where $\mathbf{n}_{B}$ is the unit normal vector on $\partial B$, pointing outward $B$. It is easy to see that its weak formulation in terms of the electric field is:

$$
\text { find } \mathbf{E}_{*} \in V: \int_{\Omega}\left(\mu^{-1} \operatorname{curl} \mathbf{E}_{*} \cdot \operatorname{curl} \overline{\mathbf{v}}+i \omega \sigma \mathbf{E}_{*} \cdot \overline{\mathbf{v}}\right)=-i \omega \int_{\partial B} \mathbf{J}_{*} \cdot \overline{\mathbf{v}} \quad \forall \mathbf{v} \in V,
$$

being $V$ the space introduced for the weak formulation of (10). Since the sesquilinear form at the left hand side is coercive, for each given $\mathbf{J}_{*} \in H^{-1 / 2}\left(\operatorname{div}_{\tau} ; \partial B\right)$ the direct problem has a unique solution.

Our first result in this section is the following.

Theorem 3.1 Assume that the coefficients $\mu$ and $\sigma$ are Lipschitz continuous and piecewise $C^{1}$ scalar functions in $\overline{\Omega_{C}}$, and that the discontinuity surfaces of their gradients are Lipschitz surfaces. Let $\left(\mathbf{E}_{*}, \mathbf{H}_{*}\right)$ be the solution of the eddy current problem driven by the surface current $\mathbf{J}_{*} \in H^{-1 / 2}\left(\operatorname{div}_{\tau} ; \partial B\right)$. The knowledge of $\mathbf{E}_{*} \times \mathbf{n}_{\Gamma}$ on $\Gamma$ uniquely determines $\mathbf{J}_{*}$. 
Proof. It is enough to show that if $\mathbf{E}_{*} \times \mathbf{n}_{\Gamma}=\mathbf{0}$ on $\Gamma$ then $\mathbf{J}_{*}=\mathbf{0}$ on $\partial B$. As in the preceding case, by solving the problem in $\Omega_{I}$ we easily show that $\mathbf{E}_{*} \times \mathbf{n}_{\Gamma}=\mathbf{0}$ on $\Gamma$ also gives $\mathbf{E}_{*}=\mathbf{0}$ in $\Omega_{I}, \mathbf{H}_{*}=\mathbf{0}$ in $\Omega_{I}$ and in particular $\mathbf{H}_{*} \times \mathbf{n}_{\Gamma}=\mathbf{0}$ on $\Gamma$. By virtue of the assumptions on the coefficients $\mu$ and $\sigma$ we can apply the unique continuation principle in $\Omega_{C} \backslash \bar{B}$ (see Ōkaji [15]) and deduce that $\mathbf{E}_{*}=\mathbf{0}$ and $\mathbf{H}_{*}=\mathbf{0}$ in $\Omega \backslash \bar{B}$.

Multiplying the second equation in (11) by a function $\mathbf{z} \in H(\operatorname{curl} ; B)$ with $\operatorname{curl}\left(\mu^{-1} \operatorname{curl} \mathbf{z}\right) \in\left(L^{2}(B)\right)^{3}$ and integrating by parts we have

$$
\int_{B} \sigma \mathbf{E}_{*} \cdot \overline{\mathbf{z}}=\int_{B} \operatorname{curl} \mathbf{H}_{*} \cdot \overline{\mathbf{z}}=-\int_{\partial B} \mathbf{H}_{* \mid B} \times \mathbf{n}_{B} \cdot \overline{\mathbf{z}}+\int_{B} \mathbf{H}_{*} \cdot \operatorname{curl} \overline{\mathbf{z}} .
$$

Since $\mathbf{H}_{*}=-(i \omega \mu)^{-1}$ curl $\mathbf{E}$, taking into account that $\mathbf{E}_{*} \times \mathbf{n}_{B}=\mathbf{0}$ on $\partial B$ another integration by parts gives

$$
\int_{B} \sigma \mathbf{E}_{*} \cdot \overline{\mathbf{z}}=-\int_{\partial B} \mathbf{H}_{* \mid B} \times \mathbf{n}_{B} \cdot \overline{\mathbf{z}}-(i \omega)^{-1} \int_{B} \mathbf{E}_{*} \cdot \operatorname{curl}\left(\mu^{-1} \operatorname{curl} \overline{\mathbf{z}}\right) .
$$

Hence for each $\mathbf{z} \in H(\operatorname{curl} ; B)$ such that $\operatorname{curl}\left(\mu^{-1} \operatorname{curl} \mathbf{z}\right)-i \omega \sigma \mathbf{z}=\mathbf{0}$ in $B$ one finds $\int_{\partial B} \mathbf{H}_{* \mid B} \times \mathbf{n}_{B} \cdot \overline{\mathbf{z}}=0$. Therefore

$$
\int_{\partial B} \mathbf{J}_{*} \cdot \overline{\mathbf{z}}=\int_{\partial B}\left[\mathbf{H}_{* \mid B} \times \mathbf{n}_{B}-\mathbf{H}_{* \mid \Omega \backslash \bar{B}} \times \mathbf{n}_{B}\right] \cdot \overline{\mathbf{z}}=0
$$

for each $\mathbf{z} \in H(\operatorname{curl} ; B)$ such that $\operatorname{curl}\left(\mu^{-1} \operatorname{curl} \mathbf{z}\right)-i \omega \sigma \mathbf{z}=\mathbf{0}$ in $B$.

Given $\boldsymbol{\rho} \in H^{-1 / 2}\left(\operatorname{curl}_{\tau} ; \Gamma\right)$, we can choose $\mathbf{z} \in H(\operatorname{curl} ; B)$, the solution to

$$
\begin{cases}\operatorname{curl}\left(\mu^{-1} \operatorname{curl} \mathbf{z}\right)-i \omega \sigma \mathbf{z}=\mathbf{0} & \text { in } B \\ \mathbf{z} \times \mathbf{n}_{B}=\boldsymbol{\rho} \times \mathbf{n}_{B} & \text { on } \partial B .\end{cases}
$$

Hence $\int_{\partial B} \mathbf{J}_{*} \cdot \overline{\boldsymbol{\rho}}=0$ for each $\boldsymbol{\rho} \in H^{-1 / 2}\left(\operatorname{curl}_{\tau} ; \Gamma\right)$, and this space is the dual space of $H^{-1 / 2}\left(\operatorname{div}_{\tau} ; \Gamma\right)$. This ends the proof.

Remark 3.1 Proceeding as in Section 2 we can obtain a representation formula similar to (8), namely,

$$
\int_{\partial B} \mathbf{J}_{*} \cdot \overline{\mathbf{z}}=(i \omega)^{-1} \int_{\Gamma} \mathbf{E}_{*} \times \mathbf{n}_{\Gamma} \cdot\left(\mu^{-1} \operatorname{curl} \overline{\mathbf{z}}\right)-\int_{\Gamma} \mathbf{H}_{*} \times \mathbf{n}_{\Gamma} \cdot \overline{\mathbf{z}}
$$

for each $\mathbf{z} \in H\left(\operatorname{curl} ; \Omega_{C}\right)$ satisfying

$$
\operatorname{curl}\left(\mu^{-1} \operatorname{curl} \mathbf{z}\right)-i \omega \sigma \mathbf{z}=\mathbf{0} \quad \text { in } \Omega_{C} .
$$

It is also possible to prove a deeper result, showing that also the discontinuity surface of $\mathbf{H}_{*} \times \mathbf{n}$ can be uniquely determined from the knowledge of the tangential component of the electric field on $\Gamma$. Suppose that $\operatorname{supp}\left(\mathbf{J}_{*}\right)=: \bar{\Sigma}$ is a connected Lipschitz surface and that $\mathbf{J}_{*} \in H_{0}^{-1 / 2}\left(\operatorname{div}_{\tau} ; \Sigma\right)$, that is, for any Lipschitz domain $B$ such that $\Sigma \subset \partial B$ it holds $\widetilde{\mathbf{J}}_{*} \in H^{-1 / 2}\left(\operatorname{div}_{\tau} ; \partial B\right)$, where $\widetilde{\mathbf{J}}_{*}$ denotes the extension of $\mathbf{J}_{*}$ by the value 0 on $\partial B \backslash \bar{\Sigma}$.

We can prove the following result. 
Theorem 3.2 Assume that the coefficients $\mu$ and $\sigma$ are Lipschitz continuous and piecewise $C^{1}$ scalar functions in $\overline{\Omega_{C}}$, and that the discontinuity surfaces of their gradients are Lipschitz surfaces. Let $\left(\mathbf{E}_{*}, \mathbf{H}_{*}\right)$ be the solution of the eddy current problem driven by the surface current $\mathbf{J}_{*} \in H_{0}^{-1 / 2}\left(\operatorname{div}_{\tau} ; \Sigma\right)$, where $\bar{\Sigma}=\operatorname{supp}\left(\mathbf{J}_{*}\right)$ is a connected Lipschitz surface. Then the knowledge of $\mathbf{E}_{*} \times \mathbf{n}_{\Gamma}$ on $\Gamma$ uniquely determines both $\Sigma$ and $\mathbf{J}_{*}$.

Proof. Let us denote by $\Sigma_{1}$ and $\Sigma_{2}$ two different surfaces where the sources $\mathbf{J}_{*, 1}$ and $\mathbf{J}_{*, 2}$ are supported, and by $\mathbf{E}_{*, 1}, \mathbf{H}_{*, 1}$ and $\mathbf{E}_{*, 2}, \mathbf{H}_{*, 2}$ the corresponding solutions, with the same value $\mathbf{E}_{*} \times \mathbf{n}$ on $\Gamma$. Solving the problem in $\Omega_{I}$ with datum $\mathbf{E}_{*} \times \mathbf{n}$ on $\Gamma$ we obtain that $\mathbf{E}_{*, 1}=\mathbf{E}_{*, 2}$ and $\mathbf{H}_{*, 1}=\mathbf{H}_{*, 2}$ in $\Omega_{I}$ and then from the unique continuation principles it follows that $\mathbf{E}_{*, 1}=\mathbf{E}_{*, 2}$ and $\mathbf{H}_{*, 1}=\mathbf{H}_{*, 2}$ in $\Omega \backslash\left(\overline{\Sigma_{1}} \cup \overline{\Sigma_{2}}\right)$.

Assume by contradiction that there exists a regular open subset of one of the two surfaces that is not contained in the other one, for instance $\widetilde{\Sigma} \subset \Sigma_{1}$ such that $\widetilde{\Sigma} \cap \Sigma_{2}=\emptyset$. Since $\widetilde{\Sigma} \subset \operatorname{supp}\left(\mathbf{J}_{*, 1}\right)$ we have that the tangential component of $\mathbf{H}_{*, 1}$ has a jump across $\widetilde{\Sigma}$ equal to $\mathbf{J}_{*, 1} \neq \mathbf{0}$. On the other hand, the tangential component of $\mathbf{H}_{*, 2}$ is continuous across $\widetilde{\Sigma}$, therefore we have reached a contradiction, as $\mathbf{H}_{*, 1}=\mathbf{H}_{*, 2}$ arbitrarily close to $\widetilde{\Sigma}$.

After having showed that $\bar{\Sigma}=\operatorname{supp}\left(\mathbf{J}_{*}\right)$ is uniquely determined, one can consider any Lipschitz domain $B$ such that $\Sigma \subset \partial B$ and extend $\mathbf{J}_{*}$ by $\mathbf{0}$ on $\partial B \backslash \bar{\Sigma}$. Denoting this extension by $\widetilde{\mathbf{J}}_{*} \in H^{-1 / 2}\left(\operatorname{div}_{\tau} ; \partial B\right)$, the unique identification of $\widetilde{\mathbf{J}}_{*}$, and thus of $\mathbf{J}_{*}$, follows from Theorem 3.1.

It is easy to verify that the same result holds if $\operatorname{supp}\left(\mathbf{J}_{*}\right)=\cup_{k=1}^{K} \overline{\Sigma_{k}}$, with $\overline{\Sigma_{k}} \cap \overline{\Sigma_{l}}=\emptyset$ for $k \neq l$ and each $\overline{\Sigma_{k}}$ is a connected Lipschitz surface.

\section{Uniqueness for dipole sources}

Let us consider now the eddy current problem with a dipole source

$$
\begin{cases}\operatorname{curl}\left(\mu^{-1} \operatorname{curl} \mathbf{E}\right)+i \omega \sigma \mathbf{E}=-i \omega \mathbf{p}_{0} \delta_{\mathbf{x}_{0}} & \text { in } \Omega \\ \operatorname{div}(\epsilon \mathbf{E})=0 & \text { in } \Omega_{I} \\ \left(\mu^{-1} \operatorname{curl} \mathbf{E}\right) \times \mathbf{n}=\mathbf{0} & \text { on } \partial \Omega \\ \epsilon \mathbf{E} \cdot \mathbf{n}=0 & \text { on } \partial \Omega,\end{cases}
$$

where $\mathbf{p}_{0} \neq \mathbf{0}, \mathbf{x}_{0} \in \Omega_{C}$ and $\delta_{\mathbf{x}_{0}}$ denotes the Dirac delta distribution centered at $\mathbf{x}_{0}$.

First we study the well-posedness of this problem. We will assume that the conductivity $\sigma$ satisfy the homogeneity condition: there exist $r_{0}>0, \mu_{0}>0$ and $\sigma_{0}>0$ such that

$$
\mu(\mathbf{x})=\mu_{0} I \text { and } \sigma(\mathbf{x})=\sigma_{0} I \text { for each } \mathbf{x} \in B_{r_{0}}\left(\mathbf{x}_{0}\right)
$$

where $I$ is the identity matrix and $B_{r_{0}}\left(\mathbf{x}_{0}\right):=\left\{\mathbf{x} \in \Omega:\left|\mathbf{x}-\mathbf{x}_{0}\right|<r_{0}\right\}$.

We set $\kappa^{2}=-i \omega \mu_{0} \sigma_{0}$ and $\mathbf{q}_{0}=-i \omega \mu_{0} \mathbf{p}_{0}$. The following result can be found in Ammari et al. [2], and we report the proof for the sake of completeness. 
Theorem 4.1 The fundamental solution $\mathbf{K}$ of the operator curl curl $-\kappa^{2} I$, that is, the solution to

$$
\operatorname{curl} \operatorname{curl} \mathbf{K}-\kappa^{2} \mathbf{K}=\mathbf{q}_{0} \delta_{\mathbf{0}}
$$

is given by

$$
\mathbf{K}(\mathbf{x})=\mathbf{q}_{0} \frac{e^{i \kappa|\mathbf{x}|}}{4 \pi|\mathbf{x}|}+\frac{1}{\kappa^{2}}\left(\mathbf{q}_{0} \cdot \operatorname{grad}\right) \operatorname{grad} \frac{e^{i \kappa|\mathbf{x}|}}{4 \pi|\mathbf{x}|} .
$$

Proof. We start from the fundamental solution $\Phi$ of the Helmholtz operator

$$
-\Delta \Phi-\kappa^{2} \Phi=\delta_{\mathbf{0}}
$$

which, as it is well-known, is given by

$$
\Phi(\mathbf{x})=\frac{e^{i \kappa|\mathbf{x}|}}{4 \pi|\mathbf{x}|} .
$$

From this we get at once

$$
-\Delta\left(\mathbf{q}_{0} \Phi\right)-\kappa^{2}\left(\mathbf{q}_{0} \Phi\right)=\mathbf{q}_{0} \delta_{\mathbf{0}}
$$

Then we look for $\mathbf{K}$ in the form

$$
\mathbf{K}=\mathbf{q}_{0} \Phi+\mathbf{q}
$$

and we have

$$
\begin{aligned}
& \text { curl curl } \mathbf{K}-\kappa^{2} \mathbf{K} \\
& \quad=-\Delta\left(\mathbf{q}_{0} \Phi\right)+\operatorname{grad} \operatorname{div}\left(\mathbf{q}_{0} \Phi\right)-\kappa^{2}\left(\mathbf{q}_{0} \Phi\right)+\operatorname{curl} \operatorname{curl} \mathbf{q}-\kappa^{2} \mathbf{q} \\
& \quad=\mathbf{q}_{0} \delta_{\mathbf{0}}+\operatorname{grad} \operatorname{div}\left(\mathbf{q}_{0} \Phi\right)+\operatorname{curl} \operatorname{curl} \mathbf{q}-\kappa^{2} \mathbf{q} .
\end{aligned}
$$

Hence $\mathbf{q}$ has to satisfy

$$
\text { curl curl } \mathbf{q}-\kappa^{2} \mathbf{q}=-\operatorname{grad} \operatorname{div}\left(\mathbf{q}_{0} \Phi\right)
$$

and we easily find

$$
\mathbf{q}=\frac{1}{\kappa^{2}} \operatorname{grad} \operatorname{div}\left(\mathbf{q}_{0} \Phi\right)
$$

In conclusion, we have obtained

$$
\begin{aligned}
\mathbf{K}(\mathbf{x}) & =\mathbf{q}_{0} \Phi(\mathbf{x})+\frac{1}{\kappa^{2}} \operatorname{grad} \operatorname{div}\left(\mathbf{q}_{0} \Phi(\mathbf{x})\right) \\
& =\mathbf{q}_{0} \frac{e^{i \kappa|\mathbf{x}|}}{4 \pi|\mathbf{x}|}+\frac{1}{\kappa^{2}}\left(\mathbf{q}_{0} \cdot \operatorname{grad}\right) \operatorname{grad} \frac{e^{i \kappa|\mathbf{x}|}}{4 \pi|\mathbf{x}|},
\end{aligned}
$$

namely, the representation formula (15).

Note that the fundamental solution $\mathbf{K}$ is much more singular than the fundamental solution of the Laplace or the Helmholtz operator: while the first term belongs to $L_{\text {loc }}^{2}$, the second one has a singularity like $|\mathbf{x}|^{-3}$. It can be also remarked that, setting $\hat{\mathbf{K}}(\mathbf{x}):=\mathbf{K}\left(\mathbf{x}-\mathbf{x}_{0}\right)$ we have $\hat{\mathbf{K}} \in H^{-2}(\Omega)$, the dual space of $H_{0}^{2}(\Omega)$; however, $\hat{\mathbf{K}}$ is a regular function far from $\mathbf{x}=\mathbf{x}_{0}$, in particular it is regular in $\overline{\Omega_{I}}$. 
Theorem 4.2 Assuming that condition (14) is satisfied, there exists a solution $\mathbf{E} \in$ $H^{-2}(\Omega)$ to (13), satisfying $(\mathbf{E}-\hat{\mathbf{K}}) \in H(\operatorname{curl} ; \Omega)$. It is unique among all the solutions $\mathbf{E}^{*}$ such that $\left(\mathbf{E}^{*}-\hat{\mathbf{K}}\right) \in H(\operatorname{curl} ; \Omega)$.

Proof. We split the solution to (13) in the following way: $\mathbf{E}(\mathbf{x})=\hat{\mathbf{K}}(\mathbf{x})+\mathbf{Q}(\mathbf{x})$. It is easily seen that we have to look for the solution $\mathbf{Q} \in H(\operatorname{curl} ; \Omega)$ to

$$
\begin{cases}\operatorname{curl}\left(\mu^{-1} \operatorname{curl} \mathbf{Q}\right)+i \omega \sigma \mathbf{Q}=\mathbf{J} & \text { in } \Omega \\ \operatorname{div}(\epsilon \mathbf{Q})=-\operatorname{div}(\epsilon \hat{\mathbf{K}}) & \text { in } \Omega_{I} \\ \left(\mu^{-1} \operatorname{curl} \mathbf{Q}\right) \times \mathbf{n}=-\left(\mu^{-1} \operatorname{curl} \hat{\mathbf{K}}\right) \times \mathbf{n} & \text { on } \partial \Omega \\ \epsilon \mathbf{Q} \cdot \mathbf{n}=-\epsilon \hat{\mathbf{K}} \cdot \mathbf{n} & \text { on } \partial \Omega\end{cases}
$$

where

$$
\mathbf{J}(\mathbf{x}):= \begin{cases}\mathbf{0} & \text { if } \mathbf{x} \in B_{r_{0}}\left(\mathbf{x}_{0}\right) \\ -\operatorname{curl}\left(\mu^{-1} \operatorname{curl} \hat{\mathbf{K}}\right)(\mathbf{x})-i \omega \sigma \hat{\mathbf{K}}(\mathbf{x}) & \text { if } \mathbf{x} \in \Omega \backslash \overline{B_{r_{0}}\left(\mathbf{x}_{0}\right)} .\end{cases}
$$

We introduce now the solution $\eta_{I} \in H^{1}\left(\Omega_{I}\right)$ of the mixed problem

$$
\begin{cases}\operatorname{div}\left(\epsilon \operatorname{grad} \eta_{I}\right)=-\operatorname{div}(\epsilon \hat{\mathbf{K}}) & \text { in } \Omega_{I} \\ \epsilon \operatorname{grad} \eta_{I} \cdot \mathbf{n}=-\epsilon \hat{\mathbf{K}} \cdot \mathbf{n} & \text { on } \partial \Omega \\ \eta_{I}=0 & \text { on } \Gamma\end{cases}
$$

which exists and is unique since $\hat{\mathbf{K}}_{\mid \Omega_{I}} \in\left(L^{2}\left(\Omega_{I}\right)\right)^{3}$; we also define

$$
\eta(\mathbf{x}):= \begin{cases}0 & \text { if } \mathbf{x} \in \Omega_{C} \\ \eta_{I}(\mathbf{x}) & \text { if } \mathbf{x} \in \Omega_{I}\end{cases}
$$

and we see at once that $\eta \in H^{1}(\Omega)$.

We are now in a position to finish the construction of the solution to (13). The solution $\mathbf{Q}$ to (16) will be found in the form $\mathbf{Q}=\mathbf{Q}^{*}+\operatorname{grad} \eta$, where $\mathbf{Q}^{*} \in H(\operatorname{curl} ; \Omega)$ is the solution to

$$
\begin{cases}\operatorname{curl}\left(\mu^{-1} \operatorname{curl} \mathbf{Q}^{*}\right)+i \omega \sigma \mathbf{Q}^{*}=\mathbf{J} & \text { in } \Omega \\ \operatorname{div}\left(\epsilon \mathbf{Q}^{*}\right)=0 & \text { in } \Omega_{I} \\ \left(\mu^{-1} \operatorname{curl} \mathbf{Q}^{*}\right) \times \mathbf{n}=-\left(\mu^{-1} \operatorname{curl} \hat{\mathbf{K}}\right) \times \mathbf{n} & \text { on } \partial \Omega \\ \epsilon \mathbf{Q}^{*} \cdot \mathbf{n}=0 & \text { on } \partial \Omega\end{cases}
$$

The existence and uniqueness of such a solution follows from the fact that the compatibility conditions

$$
\begin{aligned}
& \operatorname{div} \mathbf{J}_{\mid \Omega_{I}}=-\operatorname{div}\left[\operatorname{curl}\left(\mu^{-1} \operatorname{curl} \hat{\mathbf{K}}_{\mid \Omega_{I}}\right)\right]=0 \text { in } \Omega_{I} \\
& \mathbf{J} \cdot \mathbf{n}=-\operatorname{curl}\left(\mu^{-1} \operatorname{curl} \hat{\mathbf{K}}\right) \cdot \mathbf{n}=-\operatorname{div}_{\tau}\left(\mu^{-1} \operatorname{curl} \hat{\mathbf{K}} \times \mathbf{n}\right) \text { on } \partial \Omega
\end{aligned}
$$

are satisfied (see Alonso Rodríguez and Valli [14], Chap. 3).

We have thus found a solution $\mathbf{E}=\hat{\mathbf{K}}+\operatorname{grad} \eta+\mathbf{Q}^{*}$ of (13). Concerning uniqueness, suppose that we have another solution $\mathbf{E}^{*}$ such that $\left(\mathbf{E}^{*}-\hat{\mathbf{K}}\right) \in H(\operatorname{curl} ; \Omega)$. We can write it as $\mathbf{E}^{*}=\hat{\mathbf{K}}+\left(\mathbf{E}^{*}-\hat{\mathbf{K}}\right)$, and it is readily verified that $\mathbf{E}^{*}-\hat{\mathbf{K}}$ is a solution to (16), 
a problem for which uniqueness holds in $H(\operatorname{curl} ; \Omega)$. Therefore $\mathbf{E}^{*}-\hat{\mathbf{K}}=\mathbf{Q}=\mathbf{E}-\hat{\mathbf{K}}$, and uniqueness is proved.

Concerning the uniqueness of the inverse problem, suppose that the source is a finite sum of dipoles, in different positions and with non-vanishing polarizations, namely,

$$
\mathbf{J}_{\dagger}=\sum_{k=1}^{M} \mathbf{p}_{k} \delta_{\mathbf{x}_{k}},
$$

where $\mathbf{x}_{k} \in \Omega_{C}, \mathbf{x}_{k} \neq \mathbf{x}_{j}$ for $k \neq j, \mathbf{p}_{k} \neq \mathbf{0}$.

Theorem 4.3 Assume that $\mu$ and $\sigma$ are Lipschitz continuous and piecewise $C^{1}$ scalar functions in $\overline{\Omega_{C}}$ and that the discontinuity surfaces of their gradients are Lipschitz surfaces. Assume also that there exists the solution $\mathbf{E}_{\dagger}$ of the eddy current problem (3) driven by the surface current $\mathbf{J}_{\dagger}$ introduced in (17), with the same properties reported in Theorem 4.2. The knowledge of $\mathbf{E}_{\dagger} \times \mathbf{n}_{\Gamma}$ on $\Gamma$ uniquely determines $\mathbf{J}_{\dagger}$, namely, the number, the position and the polarization of the dipoles.

Proof. We start proving that the number and the position of the dipoles are uniquely determined.

By contradiction, let us denote by $Q_{1}$ and $Q_{2}$ two different sets of points where the dipoles are located, and by $\mathbf{E}_{\dagger, 1}, \mathbf{H}_{\dagger, 1}$ and $\mathbf{E}_{\dagger, 2}, \mathbf{H}_{\dagger, 2}$ the corresponding solutions, with the same value $\mathbf{E}_{\dagger} \times \mathbf{n}_{\Gamma}$ on $\Gamma$. As in the preceding cases, by solving the problem in $\Omega_{I}$ with datum $\mathbf{E}_{\dagger} \times \mathbf{n}_{\Gamma}$ on $\Gamma$ we obtain that $\mathbf{E}_{\dagger, 1}=\mathbf{E}_{\dagger, 2}$ and $\mathbf{H}_{\dagger, 1}=\mathbf{H}_{\dagger, 2}$ in $\Omega_{I}$.

From the unique continuation principle it follows $\mathbf{E}_{\dagger, 1}=\mathbf{E}_{\dagger, 2}$ in $\Omega \backslash\left(Q_{1} \cup Q_{2}\right)$. Let $\mathbf{x}_{*}$ a point belonging, say, to $Q_{1}$ but not to $Q_{2}$. We have that $\mathbf{E}_{\dagger, 2}$ is bounded in a neighborhood of $\mathbf{x}_{*}$, while $\mathbf{E}_{\dagger, 1}$ is unbounded there, a contradiction since $\mathbf{E}_{\dagger, 1}$ and $\mathbf{E}_{\dagger, 2}$ coincide around $\mathbf{x}_{*}$. Therefore $Q_{1}=Q_{2}$.

Let us prove now that the polarizations are uniquely determined. It is not restrictive to assume that $M=1$ and that $\mathbf{x}_{1}=\mathbf{0}$. We can write $\mathbf{E}_{\dagger, 1}=\mathbf{K}_{1}+\hat{\mathbf{E}}_{\dagger, 1}$ and $\mathbf{E}_{\dagger, 2}=\mathbf{K}_{2}+\hat{\mathbf{E}}_{\dagger, 2}$, where $\mathbf{K}_{1}$ and $\mathbf{K}_{2}$ are defined as in (15), with two different polarizations $\mathbf{q}_{0,1}$ and $\mathbf{q}_{0,2}$; in particular, we know that $\hat{\mathbf{E}}_{\dagger, 1}$ and $\hat{\mathbf{E}}_{\dagger, 2}$ belong to $H(\operatorname{curl} ; \Omega)$. Proceeding as before, the unique continuation principle yields $\mathbf{E}_{\dagger, 1}=\mathbf{E}_{\dagger, 2}$ in $\Omega \backslash B_{r}(\mathbf{0})$ for each $r>0$, therefore $\mathbf{K}_{1}-\mathbf{K}_{2}=\hat{\mathbf{E}}_{\dagger, 2}-\hat{\mathbf{E}}_{\dagger, 1}$ in $\Omega \backslash B_{r}(\mathbf{0})$. Since $\left(\hat{\mathbf{E}}_{\dagger, 2}-\hat{\mathbf{E}}_{\dagger, 1}\right) \in\left(L^{2}(\Omega)\right)^{3}$, it follows that $\mathbf{K}_{1}-\mathbf{K}_{2} \in\left(L^{2}(\Omega)\right)^{3}$, and this is not possible, due to the singularity of the fundamental solution, unless $\mathbf{K}_{1}=\mathbf{K}_{2}$, namely, $\mathbf{q}_{0,1}=\mathbf{q}_{0,2}$.

Remark 4.1 In Theorem 4.2 we have proved the existence and uniqueness of the solution $\mathbf{E}_{\dagger}$ under the homogeneity assumption (14). We have not a similar result under the assumption of Theorem 4.3.

\subsection{Explicit determination of the dipole source}

For the sake of simplicity, consider a source given by only one dipole: $\mathbf{J}_{e}=p^{*} \delta_{\mathbf{x}^{*}}$. Proceeding as in the proof of (8), one obtains the representation formula

$$
\mathbf{p}^{*} \cdot \overline{\mathbf{z}}\left(\mathbf{x}^{*}\right)=(i \omega)^{-1} \int_{\Gamma} \mathbf{E}^{*} \times \mathbf{n}_{\Gamma} \cdot\left(\mu^{-1} \operatorname{curl} \overline{\mathbf{z}}\right)-\int_{\Gamma} \mathbf{H}^{*} \times \mathbf{n}_{\Gamma} \cdot \overline{\mathbf{z}},
$$


for each $\mathbf{z} \in H\left(\operatorname{curl} ; \Omega_{C}\right)$, continuous around $\mathbf{x}^{*}$ and satisfying

$$
\operatorname{curl}\left(\mu^{-1} \operatorname{curl} \mathbf{z}\right)-i \omega \sigma \mathbf{z}=\mathbf{0} \text { in } \Omega_{C} .
$$

To determine the source, we have to find the polarization $\mathbf{p}^{*}$ and the position $\mathbf{x}^{*}$ : therefore, six parameters. The natural idea is to choose in a suitable way six functions $\mathbf{z}$ in (18), and solve the corresponding nonlinear system.

Let us assume that $\mu$ and $\sigma$ are constants. The usual choice is to take $\mathbf{z}(\mathbf{x})=\mathbf{b} e^{i \kappa \mathbf{d} \cdot \mathbf{x}}$, with $\kappa \in \mathbb{C}, \mathbf{b} \in \mathbb{R}^{3}, \mathbf{d} \in \mathbb{R}^{3}$. In order that $\mathbf{z}$ is a solution to (19) we need

$$
\kappa^{2}=i \omega \mu \sigma \quad, \quad \mathbf{b} \cdot \mathbf{d}=0 .
$$

hence $\kappa=\sqrt{\frac{\omega \mu \sigma}{2}}(1+i)$ if $\omega>0$ and $\kappa=\sqrt{\frac{|\omega \mu \sigma|}{2}}(1-i)$ if $\omega<0$. It is not restrictive to assume $|\mathbf{d}|=|\mathbf{b}|=1$.

The values of $\mathbf{p}^{*}$ and $\mathbf{x}^{*}$ are uniquely determined by solving the nonlinear system (18) obtained by suitable selections of $\mathbf{b}$ and $\mathbf{d}$. For instance taking $\mathbf{b}=\mathbf{e}_{1}, \mathbf{d}=\mathbf{e}_{2}$, or $\mathbf{b}=\mathbf{e}_{2}, \mathbf{d}=\mathbf{e}_{3}$, or $\mathbf{b}=\mathbf{e}_{3}, \mathbf{d}=\mathbf{e}_{2}$, where $\mathbf{e}_{i}$ represents the standard euclidean basis, one has

$$
-i \omega p_{1}^{*} e^{-i \bar{\kappa} x_{2}^{*}}=A_{1,2}, \quad-i \omega p_{2}^{*} e^{-i \bar{\kappa} x_{3}^{*}}=A_{2,3}, \quad-i \omega p_{3}^{*} e^{-i \bar{\kappa} x_{2}^{*}}=A_{3,2} .
$$

Here $A_{i, j}$ denotes the right hand side in equation (18), hence a computable complex number, corresponding to the choice $\mathbf{z}(\mathbf{x})=\mathbf{b} e^{i \kappa \mathbf{d} \cdot \mathbf{x}}$, with $\mathbf{b}=\mathbf{e}_{i}, \mathbf{d}=\mathbf{e}_{j}$. Since $\mathbf{p}^{*} \neq \mathbf{0}$, at least one of its components is different from 0 , hence at least one of the values $A_{1,2}$, $A_{2,3}$ or $A_{3,2}$ is different from 0 . Let us assume for instance that $p_{1}^{*} \neq 0, A_{1,2} \neq 0$. Taking $\mathbf{b}=\mathbf{e}_{1}, \mathbf{d}=\mathbf{e}_{3}$ and $\mathbf{b}=\mathbf{e}_{1}, \mathbf{d}=\frac{1}{\sqrt{2}}\left(\mathbf{e}_{2}+\mathbf{e}_{3}\right)$ one has

$$
-i \omega p_{1}^{*} e^{-i \bar{\kappa} x_{3}^{*}}=A_{1,3}, \quad-i \omega p_{1}^{*} e^{-i \bar{\kappa} \frac{1}{\sqrt{2}}\left(x_{2}^{*}+x_{3}^{*}\right)}=A_{1,23},
$$

where $A_{1,23}$ is obtained as before from the latter choice of $\mathbf{b}$ and $\mathbf{d}$. Hence

$$
e^{-i \bar{\kappa}\left(x_{3}^{*}-x_{2}^{*}\right)}=\frac{A_{1,3}}{A_{1,2}}, \quad e^{-i \bar{\kappa}\left[\frac{1}{\sqrt{2}}\left(x_{2}^{*}+x_{3}^{*}\right)-x_{2}^{*}\right]}=\frac{A_{1,23}}{A_{1,2}},
$$

For the sake of simplicity let us assume $\omega>0$, so that $-i \bar{\kappa}=-\sqrt{\frac{\omega \mu \sigma}{2}}(1+i)$ and

$$
\begin{aligned}
& \left|e^{-i \bar{\kappa}\left(x_{3}^{*}-x_{2}^{*}\right)}\right|=e^{-\sqrt{\frac{\omega \mu \sigma}{2}}\left(x_{3}^{*}-x_{2}^{*}\right)}=\left|\frac{A_{1,3}}{A_{1,2}}\right| \\
& \left|e^{-i \bar{\kappa}\left[\frac{1}{\sqrt{2}}\left(x_{2}^{*}+x_{3}^{*}\right)-x_{2}^{*}\right]}\right|=e^{-\sqrt{\frac{\omega \mu \sigma}{2}}\left[\frac{1}{\sqrt{2}}\left(x_{2}^{*}+x_{3}^{*}\right)-x_{2}^{*}\right]}=\left|\frac{A_{1,23}}{A_{1,2}}\right| .
\end{aligned}
$$

Therfore one has the following linear system for $x_{2}^{*}$ and $x_{1}^{*}$

$$
\begin{aligned}
& x_{3}^{*}-x_{2}^{*}=-\sqrt{\frac{2}{\omega \mu \sigma}} \log \left|\frac{A_{1,3}}{A_{1,2}}\right|, \\
& \frac{1}{\sqrt{2}}\left(x_{2}^{*}+x_{3}^{*}\right)-x_{2}^{*}=-\sqrt{\frac{2}{\omega \mu \sigma}} \log \left|\frac{A_{1,23}}{A_{1,2}}\right|,
\end{aligned}
$$

obtaining

$$
\begin{aligned}
& x_{2}^{*}=\frac{\sqrt{2}+1}{\sqrt{\omega \mu \sigma}}\left[\log \left|\frac{A_{1,3}}{A_{1,2}}\right|-\sqrt{2}\left|\frac{A_{1,23}}{A_{1,2}}\right|\right] \\
& x_{3}^{*}=\frac{1}{\sqrt{\omega \mu \sigma}}\left[\log \left|\frac{A_{1,3}}{A_{1,2}}\right|-(2+\sqrt{2})\left|\frac{A_{1,23}}{A_{1,2}}\right|\right] .
\end{aligned}
$$


Replacing in (20) we can compute the three components of $\mathbf{p}^{*}$.

It remains to compute $x_{1}^{*}$. If $\mathbf{p}^{*}$ has two components different from zero, say, $p_{1}^{*} \neq 0$ and $p_{2}^{*} \neq 0$, taking $\mathbf{b}=\mathbf{e}_{2}$ and $\mathbf{d}=\mathbf{e}_{1}$ we have the equation

$$
-i \omega p_{2}^{*} e^{-i \bar{\kappa} x_{1}^{*}}=A_{2,1}
$$

from which we determine $x_{1}^{*}$. Otherwise, taking $\mathbf{b}=\frac{1}{\sqrt{2}}\left(\mathbf{e}_{1}+\mathbf{e}_{2}\right)$ and $\mathbf{d}=\frac{1}{\sqrt{2}}\left(\mathbf{e}_{1}-\mathbf{e}_{2}\right)$ we obtain

$$
-i \omega \frac{1}{\sqrt{2}} p_{1}^{*} e^{-i \bar{\kappa} \frac{1}{\sqrt{2}}\left(x_{1}^{*}-x_{2}^{*}\right)}=A_{12,12},
$$

with the usual notation for $A_{12,12}$.

\section{Application to EEG/MEG}

Magnetoencephalography measures the magnetic induction along a certain direction depending of the magnetometer. Typically it measures the normal component of the magnetic induction, namely, $\mu \mathbf{H} \cdot \mathbf{n}_{\Gamma}$. On the other hand, electroencephalography measures the electric potential on the surface of the head. We have obtained some explicit relations between the source and the tangential component of the electric field and the magnetic field on $\Gamma$ (equations (8), (12), and (18)). We will show that, when using the eddy current model, both $\left(\mathbf{E} \times \mathbf{n}_{\Gamma}\right)_{\mid \Gamma}$ and $\left(\mathbf{H} \times \mathbf{n}_{\Gamma}\right)_{\mid \Gamma}$ can be computed from the normal component of the magnetic induction $\left(\mu \mathbf{H} \cdot \mathbf{n}_{\Gamma}\right)_{\mid \Gamma}$ and the electric potential $V_{\mid \Gamma}$.

First of all, notice that in the eddy current model, if we are given with $\left(\mu \mathbf{H} \cdot \mathbf{n}_{\Gamma}\right)_{\mid \Gamma}$, the magnetic field in the insulator is the unique solution of

$$
\begin{cases}\operatorname{curl} \mathbf{H}_{I}=\mathbf{0} & \text { in } \Omega_{I} \\ \operatorname{div}\left(\mu \mathbf{H}_{I}\right)=0 & \text { in } \Omega_{I} \\ \mu \mathbf{H}_{I} \cdot \mathbf{n}_{\Gamma}=\mu \mathbf{H} \cdot \mathbf{n}_{\Gamma} & \text { on } \Gamma \\ \mathbf{H}_{I} \times \mathbf{n}=\mathbf{0} & \text { on } \partial \Omega \\ \mathbf{H}_{I} \perp \mathcal{H}_{\mu}\left(\partial \Omega, \Gamma ; \Omega_{I}\right), & \end{cases}
$$

where $\mathcal{H}_{\mu}\left(\partial \Omega, \Gamma ; \Omega_{I}\right)$ denotes the space of harmonic fields

$$
\begin{aligned}
\mathcal{H}_{\mu}\left(\partial \Omega, \Gamma ; \Omega_{I}\right):=\left\{\mathbf{v}_{I} \in\left(L^{2}\left(\Omega_{I}\right)\right)^{3} \mid \operatorname{curl} \mathbf{v}_{I}=\mathbf{0}, \operatorname{div}\left(\mu \mathbf{v}_{I}\right)=0,\right. \\
\left.\mathbf{v}_{I} \times \mathbf{n} \text { on } \partial \Omega, \mu \mathbf{v}_{I} \cdot \mathbf{n}_{\Gamma} \text { on } \Gamma\right\} .
\end{aligned}
$$

Hence $\left(\mathbf{H} \times \mathbf{n}_{\Gamma}\right)_{\mid \Gamma}$ can be computed if we know $\left(\mu \mathbf{H} \cdot \mathbf{n}_{\Gamma}\right)_{\mid \Gamma}$.

Now we need some preliminaries concerning tangential differential operators. The standard definition of the tangential gradient and the tangential curl on the flat surface $\left\{x_{3}=0\right\}$ with unit normal vector $\mathbf{n}=(0,0,1)$ is

$$
\operatorname{grad}_{\tau} \phi=\left(\partial_{1} \phi, \partial_{2} \phi, 0\right) \quad \operatorname{Curl}_{\tau} \phi=\operatorname{grad}_{\tau} \phi \times \mathbf{n}=\left(\partial_{2} \phi,-\partial_{1} \phi, 0\right) .
$$

Using local coordinates it is possible to define the operators $\operatorname{grad}_{\tau}$ and $\mathrm{Curl}_{\tau}$ for function belonging to $H^{1}(\Gamma)$. By a duality argument the adjoint operators $\operatorname{div}_{\tau}$ and $\operatorname{curl}_{\tau}$ are also introduced, as well as the Laplace-Beltrami operator $\Delta_{\tau}:=\operatorname{div}_{\tau} \operatorname{grad}_{\tau}=-\operatorname{curl}_{\tau} \operatorname{Curl}_{\tau}$. 
On $\Gamma$ one has the following Hodge decomposition of the electric field (see Buffa et al. [16]):

$$
\mathbf{n}_{\Gamma} \times \mathbf{E} \times \mathbf{n}_{\Gamma}=\operatorname{grad}_{\tau} v+\operatorname{Curl}_{\tau} q,
$$

with $q \in H^{1}(\Gamma) / \mathbb{C}$ such that

$$
\begin{aligned}
\Delta_{\tau} q & =-\operatorname{curl}_{\tau} \operatorname{Curl}_{\tau} q=-\operatorname{curl}_{\tau}\left(\mathbf{n}_{\Gamma} \times \mathbf{E} \times \mathbf{n}_{\Gamma}\right) \\
& =-\operatorname{div}_{\tau}\left(\mathbf{E} \times \mathbf{n}_{\Gamma}\right)=-\operatorname{curl} \mathbf{E} \cdot \mathbf{n}_{\Gamma}=i \omega \mu \mathbf{H} \cdot \mathbf{n}_{\Gamma},
\end{aligned}
$$

and $v \in H^{1 / 2}(\Gamma), v=V_{\mid \Gamma}$ with $V \in H^{1}\left(\Omega_{C}\right)$ and $\operatorname{grad}_{\tau} v=\mathbf{n}_{\Gamma} \times \operatorname{grad} V \times \mathbf{n}_{\Gamma}$. Hence $\mathbf{n}_{\Gamma} \times \mathbf{E} \times \mathbf{n}_{\Gamma}$ can be obtained from the knowledge of $\mu \mathbf{H} \cdot \mathbf{n}_{\Gamma}$ and $V$ on $\Gamma$.

Remark 5.1 Though it could sound strange, it is not completely clear what we say when we speak about the measure of the scalar electric potential (see, e.g., Nicholson [17], Bossavit [18], and the references therein). In fact, with the exception of the static case, the electric field is not irrotational, therefore it has not a scalar potential.

As it is well-known, the electric field $\mathbf{E}$ can be split into the sum of a gradient and a solenoidal field, but this can be done in several different way (see, e.g., Alonso Rodríguez and Valli [14], Sect. A.3). Hence, here we are saying that, if the measure obtained by a voltmeter is the scalar $v$ appearing in (22) (up to an additive constant) and the measure obtained by a magnetometer is $\mu \mathbf{H} \cdot \mathbf{n}_{\Gamma}$, then we can reconstruct the value of $\mathbf{n}_{\Gamma} \times \mathbf{E} \times \mathbf{n}_{\Gamma}$ on $\Gamma$.

In real-life applications the measurements are only made on a subset of the boundary $\Gamma_{m} \subset \Gamma$. Also in this case it is possible to obtain a representation formula for the source in term of the tangential components of the electric and the magnetic fields on $\Gamma_{m}$. Following Albanese and Monk [3] it is easy to show formally that for any $\mathbf{z} \in H\left(\operatorname{curl} ; \Omega \backslash \Gamma_{m}\right)$ such that $\operatorname{curl}\left(\mu^{-1} \operatorname{curl} \mathbf{z}\right)-i \omega \sigma \mathbf{z}=\mathbf{0}$ in $\Omega \backslash \Gamma_{m}$ and $\left(\mu^{-1} \operatorname{curl} \mathbf{z}\right) \times \mathbf{n}=\mathbf{0}$ on $\partial \Omega$, we have

$$
\left\langle\mathbf{J}_{e}, \mathbf{z}\right\rangle=(i \omega)^{-1} \int_{\Gamma_{m}} \mathbf{E} \times \mathbf{n}_{\Gamma} \cdot\left[\left[\mu^{-1} \operatorname{curl} \overline{\mathbf{z}}\right]\right]_{T}-\int_{\Gamma_{m}} \mathbf{H} \times \mathbf{n}_{\Gamma} \cdot[[\overline{\mathbf{z}}]]_{T},
$$

where $[[\mathbf{v}]]_{T}$ denotes the jump of the tangential trace of $\mathbf{v} \in H\left(\operatorname{curl} ; \Omega \backslash \Gamma_{m}\right)$ across $\Gamma_{m}$ and $\left\langle\mathbf{J}_{e}, \mathbf{z}\right\rangle=\int_{\Omega_{C}} \mathbf{J}_{e} \cdot \overline{\mathbf{z}}$ for volume currents $\mathbf{J}_{e} \in\left(L^{2}\left(\Omega_{C}\right)\right)^{3},\left\langle\mathbf{J}_{e}, \mathbf{z}\right\rangle=\int_{\partial B} \mathbf{J}_{e} \cdot \overline{\mathbf{z}}$ for surface currents $\mathbf{J}_{e} \in H^{-1 / 2}\left(\operatorname{div}_{\tau} ; \partial B\right)$, and $\left\langle\mathbf{J}_{e}, \mathbf{z}\right\rangle=\mathbf{p}^{*} \cdot \overline{\mathbf{z}}\left(\mathbf{x}^{*}\right)$ for a dipole source $\mathbf{J}_{e}=\mathbf{p}^{*} \delta_{\mathbf{x}^{*}}$.

Also in this case the tangential component of the electric field on $\Gamma_{m}$ can be obtained form the electric potential and the normal component of the magnetic induction, provided that the measured electric potential $V$ is such that

$$
\operatorname{grad} V \cdot \mathbf{t}=\mathbf{E} \cdot \mathbf{t} \quad \text { on } \partial \Gamma_{m},
$$

where $\mathbf{t}$ is the unit tangent vector on $\partial \Gamma_{m}$. In this way $q \in H^{1}\left(\Gamma_{m}\right) / \mathbb{C}$ is the solution of

$$
\begin{cases}\Delta_{\tau} q=i \omega \mu \mathbf{H} \cdot \mathbf{n}_{\Gamma} & \text { in } \Gamma_{m} \\ \operatorname{Curl}_{\tau} q \cdot \mathbf{t}=0 & \text { on } \partial \Gamma_{m} .\end{cases}
$$

However, if we know $\mathbf{E} \times \mathbf{n}_{\Gamma}$ only on $\Gamma_{m}$ and not in the whole boundary $\Gamma$, it is not possible to obtain $\left(\mathbf{H} \times \mathbf{n}_{\Gamma}\right)_{\mid \Gamma_{m}}$ from (21). So in order to use the representation formula (23) in an inversion scheme it would be necessary to measure also the tangential component of the magnetic field on $\Gamma_{m}$. 


\section{References}

[1] S. He and V. G. Romanov. Identification of dipole sources in a bounded domain for Maxwell's equations. Wave Motion, 28(1):25-40, 1998.

[2] H. Ammari, G. Bao, and J. L. Fleming. An inverse source problem for Maxwell's equations in magnetoencephalography. SIAM J. Appl. Math., 62(4):1369-1382, 2002.

[3] R. Albanese and P. B. Monk. The inverse source problem for Maxwell's equations. Inverse Problems, 22(3):1023-1035, 2006.

[4] J. Sarvas. Basic mathematical and electromagnetic concepts of the biomagnetic inverse problem. Physics in Medicine and Biology, 32(1):11-22, 1987.

[5] R. Kress, L. Kühn, and R. Potthast. Reconstruction of a current distribution from its magnetic field. Inverse Problems, 18(4):1127-1146, 2002.

[6] A. S. Fokas, Y. Kurylev, and V. Marinakis. The unique determination of neuronal currents in the brain via magnetoencephalography. Inverse Problems, 20(4):1067-1082, 2004.

[7] G. Dassios and D. Hadjiloizi. On the non-uniqueness of the inverse problem associated with electroencephalography. Inverse Problems, 25(11):115012, 18, 2009.

[8] A. El Badia and T. Nara. An inverse source problem for quasi-static Maxwell's equations. J. Inverse Ill-Posed Probl., 18(7):741-764, 2010.

[9] N. Bleistein and J. K. Cohen. Nonuniqueness in the inverse source problem in acoustics and electromagnetics. J. Mathematical Phys., 18(2):194-201, 1977.

[10] S. Andrieux and A. Ben Abda. Identification of planar cracks by complete overdetermined data: inversion formulae. Inverse Problems, 12(5):553-563, 1996.

[11] A. El Badia and T. Ha Duong. Some remarks on the problem of source identification from boundary measurements. Inverse Problems, 14(4):883-891, 1998.

[12] P. S. Novikov. Sur le problème inverse du potentiel. Doklady Akad. Nauk SSSR (N.S.), 18:165-168, 1938.

[13] V. Isakov. Inverse Source Problems, volume 34 of Mathematical Surveys and Monographs. American Mathematical Society, Providence, RI, 1990.

[14] A. Alonso Rodríguez and A. Valli. Eddy Current Approximation of Maxwell Equations, volume 4 of MSEA. Modeling, Simulation and Applications. Springer-Verlag Italia, Milan, 2010.

[15] T. Ōkaji. Strong unique continuation property for time harmonic Maxwell equations. J. Math. Soc. Japan, 54(1):89-122, 2002.

[16] A. Buffa, M. Costabel, and D. Sheen. On traces for $\mathbf{H}(\mathbf{c u r l}, \Omega)$ in Lipschitz domains. J. Math. Anal. Appl., 276(2):845-867, 2002.

[17] H.W. Nicholson. What does the voltmeter read? Am. J. Phys., 73(12):1194-1196, 2005.

[18] A. Bossavit. What do voltmeters measure? COMPEL, 27(1):9-16, 2008. 